Histoire de l'éducation

\title{
Entre histoire croisée et histoire dénationalisée. Le manuel franco-allemand d'histoire
}

Betwixt Hybrid and Non-National History. The Franco-German History Textbook Geschichte über Kreuz und ohne nationale Bindung. Das deutsch-französische Geschichtsbuch

Entre historia cruzada e historia desnacionalizada. El manual franco-alemán de historia

\section{Emmanuel Droit}

\section{OpenEdition}

Journals

Édition électronique

URL : https://journals.openedition.org/histoire-education/1251

DOI : 10.4000/histoire-education. 1251

ISSN : 2102-5452

Éditeur

ENS Éditions

Édition imprimée

Date de publication : 1 mai 2007

Pagination : 151-162

ISBN : 978-2-7342-1095-5

ISSN : 0221-6280

\section{Référence électronique}

Emmanuel Droit, «Entre histoire croisée et histoire dénationalisée. Le manuel franco-allemand d'histoire », Histoire de l'éducation [En ligne], 114 | 2007, mis en ligne le 01 janvier 2012, consulté le 20 mai 2021. URL : http://journals.openedition.org/histoire-education/1251 ; DOI : https://doi.org/ 10.4000/histoire-education. 1251 


\section{ENTRE HISTOIRE CROISÉE ET HISTOIRE DÉNATIONALISÉE Le manuel franco-allemand d'histoire}

par Emmanuel DROIT

Depuis le XIX ${ }^{\mathrm{e}}$ siècle et la construction des États-nations, l'histoire est considérée comme un des fondements de l'identité nationale et un des vecteurs du nationalisme, tant par la façon dont elle est pratiquée que par celle dont elle est enseignée (1). Les manuels scolaires constituent dans le champ scolaire des vecteurs culturels reflétant les intentions éducatives de tel ou tel régime politique (2). Or, depuis la rentrée scolaire 2006, enseignants et élèves français et allemands des classes terminales (classe de terminale en France, $12^{\mathrm{e}}$ ou $13^{\mathrm{e}}$ classe, selon le Land, en Allemagne) ont à leur disposition un manuel commun d'histoire sur l'Europe et le monde depuis 1945 au contenu rigoureusement identique (3). Il s'agit d'un manuel franco-allemand d'histoire et non d'un manuel d'histoire franco-allemande (4). Le rappel de cette distinction vise à souligner toute l'ambition de ce projet éditorial original: écrire une histoire commune du monde, de l'Europe, de la France et de l'Allemagne (RFA et RDA) depuis 1945 tout en respectant la diversité des interprétations de tel ou tel phénomène historique et en synthétisant les approches didactiques pratiquées de part et d'autre du

(1) Voir à ce sujet Pierre Nora, «L'histoire de France de Lavisse. Pietas erga patriam », in Pierre Nora (dir.), Les Lieux de mémoire, t. 2: La Nation. Héritage, historiographie, paysages, Paris, Gallimard, 1986, pp. 317-376; Anne-Marie Thiesse, La création des identités nationales, Paris, Seuil, 1999.

(2) Jeannie Bauvois-Cauchepin, Enseignement de l'histoire et mythologie nationale. Allemagne-France du début du XX $X^{e}$ siècle aux années 1950, Berne, Peter Lang, 2002.

(3) Peter Geiss, Guillaume Le Quintrec (dir.), Histoire/Geschichte: l'Europe et le monde depuis 1945, manuel d'histoire franco-allemand. Terminales L/ES/S, Paris, Nathan, 2006.

(4) Pierre Monnet, «Un manuel d'histoire franco-allemand », Revue historique, $\mathrm{n}^{\circ} 639,2006$, pp. 409-422. 
Rhin. L'historien français Étienne François, une des têtes pensantes de ce manuel, parle à juste titre de « saut qualitatif de première importance dans l'enseignement de l'histoire » (1).

Cette entreprise inédite constitue tout à la fois l'aboutissement d'un projet dont les prodromes remontent à l'entre-deux-guerres et le point de départ d'une nouvelle manière d'enseigner l'histoire dans le secondaire. Dans quelle mesure le manuel franco-allemand d'histoire constitue-t-il le " nouveau fleuron d'un modèle de réconciliation réussie entre deux ennemis héréditaires » (2)? Quelles sont les caractéristiques scientifiques et didactiques qui font sa spécificité par rapport aux manuels classiques franco-français?

À travers cette contribution, nous entendons d'une part mettre en perspective l'historicité du manuel franco-allemand en retraçant sa genèse dans un cadre chronologique large, et d'autre part présenter son caractère novateur, en soulignant notamment l'intérêt de l'enseignement d'une histoire dénationalisée et croisée avec celle d'une autre nation, en phase avec le temps de la mondialisation.

\section{LA GENÈSE DU MANUEL FRANCO-ALLEMAND D'HISTOIRE}

Si la parution de cet ouvrage est chose récente, l'idée qui a présidé à sa production est bien plus ancienne. La longue durée de la période nécessaire à sa réalisation témoigne à la fois de la précocité des aspirations à la paix entre la France et l'Allemagne et des obstacles qui ont longtemps retardé celle-ci.

\section{Le manuel d'histoire comme produit de la réconciliation franco-allemande}

Les discussions sur la possibilité de concevoir un manuel francoallemand d'histoire remontent au début des années 1930 et s'inscri-

(1) Étienne François, « Le manuel franco-allemand d'histoire. Une entreprise inédite », Vingtième Siècle. Revue d'histoire, n 94, avril-juin 2007, pp. 73-86.

(2) Corinne Defrance, Ulrich Pfeil, «Au service du rapprochement franco-allemand. Dialogues d'historiens de part et d'autre du Rhin », in Georges Mink, Laure Neumayer (dir.), L'Europe et ses passés douloureux, Paris, La Découverte, 2007, «Recherches », p. 101. 
vent plus globalement dans un contexte de tentative de révision des manuels scolaires que le mouvement pacifiste (notamment les partis socialistes) appelle de ses vœux dès les années 1880 . Elles s'inscrivent en outre dans le prolongement de la réaction morale à la façon dont l'histoire fut instrumentalisée lors de la Première Guerre mondiale pour la «mobilisation des esprits ».

Au lendemain de 1918, des instituteurs, des pédagogues et des historiens, soutenus, entre autres, par la Fondation Carnegie et la Société des Nations (SDN), réfléchissent à la façon dont les manuels scolaires peuvent être révisés, l'objectif étant d'éliminer de l'enseignement historique les stéréotypes nationaux pour contribuer au « désarmement moral » des nouvelles générations (1). En 1923, la Commission Internationale de Coopération Intellectuelle (CICI), qui œuvre sous l'autorité de la SDN, a même élaboré un projet de manuel unique pour tous les pays. L'idée est reprise en 1929 par le Comité international des Sciences Historiques, mais elle n'a jamais dépassé le stade de la réflexion. En 1931, le médiéviste allemand Fritz Kern et l'intellectuel français Jean de Pange proposent un projet de manuel commun des relations franco-allemandes de l'époque franque à la Première Guerre mondiale, en trois volumes. Mais ce «plan Kellog de la science » (2) présenté à la conférence internationale pour l'enseignement de l'histoire de La Haye en 1932 ne survit pas à la dégradation du climat politique liée à l'accession d'Hitler au pouvoir.

La faillite du manuel commun s'est doublée de l'échec de la tentative franco-allemande de réviser les manuels scolaires. Autorisée par le régime nazi à des seules fins de respectabilité, la rencontre entre enseignants d'histoire des deux pays organisée à Paris en 1935 s'est achevée par un communiqué final présentant trente-neuf thèses visant à améliorer l'enseignement de l'histoire de l'époque moderne et contemporaine. Mais cet appel est resté lettre morte sous le Troisième Reich, qui, au contraire, s'est attaché avec la publication de programmes nationaux en 1938 à valoriser une conception raciste et

(1) Maria Cristina Giuntella, «Enseignement de l'histoire et révision des manuels scolaires dans l'entre-deux-guerres », in Marie-Christine Baquès, Annie Bruter et Nicole Tutiaux-Guillon (dir.), Pistes didactiques et chemins d'historiens. Textes offerts à Henri Moniot, Paris, L'Harmattan, 2003, pp. 161-189.

(2) Corinne Defrance, Ulrich Pfeil, «Au service du rapprochement franco-allemand. Dialogues d'historiens de part et d'autre du Rhin », op. cit., p. 99. 
nationaliste de l'histoire (1). Quoiqu'insuffisants et inefficaces, ces projets franco-allemands inachevés ont posé les jalons du principe de rencontres bilatérales.

Au lendemain de la Seconde Guerre mondiale, l'idée de réconciliation franco-allemande est mise en pratique par des historiens et enseignants français et allemands. Ce dialogue régulier, lancé entre autres par Georg Eckert (futur directeur de l'Institut international de recherches sur les manuels scolaires de Brunswick), débouche sur la volonté d'améliorer concrètement l'enseignement de l'histoire du pays voisin, c'est-à-dire de clarifier le problème des caractères nationaux et de mettre l'accent sur les liens qui unissent les deux pays (la figure de Charlemagne, les relations franco-allemandes à l'époque médiévale). En mettant l'accent sur le dialogue et la coopération scientifique, les historiens français et allemands de l'après-guerre ont préparé le terrain en « nettoyant » les manuels d'histoire de la mythologie nationale et en travaillant à l'élaboration d'une explication consensuelle et apaisée du passé. Ils ont été les acteurs essentiels de la constitution et de la diffusion d'une "grammaire de réconciliation » qui a contribué au rapprochement des anciens « ennemis héréditaires ».

\section{Une initiative de la société civile transformée en projet politique majeur}

Le manuel franco-allemand d'histoire s'inscrit dans le contexte particulier de la célébration de la réconciliation réussie entre la France et l'Allemagne depuis 1945. À l'occasion du quarantième anniversaire du traité d'amitié franco-allemand, en janvier 2003, les autorités des deux pays ont réuni à Berlin un Parlement des jeunes. Ce dernier, organisé par l'Office franco-allemand pour la jeunesse, avait pour objectif de faire participer 500 jeunes des deux pays (250 élèves français et 250 élèves allemands âgés de 17 à 19 ans) au débat public sur des questions politiques d'intérêt général, et en particulier sur leurs attentes vis-à-vis de la construction européenne et du rôle à jouer par la France et l'Allemagne dans son approfondissement. Ces jeunes appartiennent à une génération qui n'a pas connu la guerre et qui a grandi dans l'esprit de réconciliation. Lors de la session plénière tenue en présence du Président Chirac et du Chancelier

(1) Jeannie Bauvois-Cauchepin, op. cit. 
Schröder, l'un des souhaits formulés par ce Parlement des jeunes a été « l'introduction d'un manuel d'histoire avec les mêmes contenus pour les deux pays afin de réduire les préjugés causés par la méconnaissance mutuelle et de dépasser une histoire marquée par plusieurs guerres meurtrières entre l'Allemagne et la France »(1). Cette demande illustre le succès des politiques de réconciliation, entendues comme apaisement des tensions interétatiques entre les deux pays, et menées tant au niveau de la société civile que des instances gouvernementales. Elle correspond aussi en partie à un véritable besoin, exprimé par des participants fréquentant les classes européennes et AbiBac (2), qui se sont fortement développées depuis le milieu des années 1990. Enfin, elle exprime la volonté de dépasser le cadre national et de favoriser non seulement la compréhension mutuelle entre les deux peuples, mais aussi l'émergence d'une conscience européenne commune, sur les bases d'une histoire apaisée, véritable antidote aux nationalismes.

Cette initiative a immédiatement reçu le soutien explicite des chefs de l'exécutif des deux gouvernements, qui étaient à la recherche d'une initiative destinée à donner un souffle nouveau au couple franco-allemand, en perte de vitesse depuis la réunification allemande. Ils souhaitaient aussi marquer leur passage au pouvoir par une réalisation inédite et forte sur le plan symbolique: l'ambition était de montrer que la relation franco-allemande n'est pas seulement une réalité politique, mais également une réalité pédagogique. Ce projet, issu au départ d'une demande de la société civile, a donc été repris en compte par les instances gouvernementales des deux pays, qui vont soigneusement veiller à ce qu'il aboutisse dans un délai extrêmement

(1) Parlement franco-allemand des jeunes, Déclaration finale sur l'avenir des relations franco-allemandes. Voir le texte sur le site de l'OFAJ, http://www.ofaj.org/ fr/ofaj/40ans/pjresolution.htm.

(2) Créées en 1992, les sections européennes ont pour vocation de proposer un enseignement ouvert sur les pays étrangers par l'apprentissage renforcé d'une langue étrangère, l'enseignement en langue étrangère d'une discipline non linguistique et la connaissance approfondie de la culture du pays de la langue en question. Conçues dans le cadre de la coopération franco-allemande en 1987, formalisées par le traité de Mulhouse de 1994, les sections AbiBac sont des sections bilingues à profil franco-allemand fondées sur le jumelage d'un établissement français et d'un établissement allemand et sur la conception commune de projets qui trouvent leur réalisation au cours de contacts réguliers et d'échanges. Les établissements accueillant l'AbiBac dispensent à partir de la classe de seconde une formation qui prépare à la double délivrance du baccalauréat général et de l'Abitur. 
rapide. Elles mettent en place un comité de pilotage paritaire francoallemand, constitué d'universitaires et de représentants des institutions politiques et éducatives, qui sont chargés d'établir un cahier des charges, c'est-à-dire de définir précisément les thèmes qui vont constituer les chapitres des trois volumes du futur manuel. Le projet est en effet subdivisé en trois parties : un premier volume consacré à la période postérieure à la Seconde Guerre mondiale, un second couvrant la période allant de 1815 à 1945 (1), et un troisième volume traitant la période allant de l'Antiquité au Congrès de Vienne.

Au cours de leurs nombreuses réunions, les membres du comité de pilotage ont pu se rendre compte que les divergences de fond étaient minimes et qu'elles pouvaient rapidement être surmontées. Cet état de choses est le fruit d'une tradition déjà ancienne d'échanges et de collaboration entre historiens français et allemands, initiée entre 1948 et 1950 par Raymond Schmittlein, directeur de l'Éducation publique du gouvernement militaire français à BadenBaden, lors des « rencontres de Spire » (2). Il est intéressant de noter que le comité de pilotage s'est divisé essentiellement sur deux questions dont on n'imaginait pas a priori qu'elles posent problème, à savoir celle des invasions barbares et celle du rôle des États-Unis dans l'évolution de l'Europe après 1945. La première pomme de discorde peut surprendre, dans la mesure où le Moyen Âge est une période qui ne semble guère de nature à susciter de controverses au regard de l'histoire la plus contemporaine. Pourtant, des difficultés sont apparues en rapport avec l'utilisation du concept de "Barbares », jugé scandaleux par les Allemands, qui parlent plutôt de « migrations de peuples » (Völkerwanderungen en allemand) (3). Le second point de friction tient au fait que les Allemands désiraient mettre l'accent sur le rôle positif des États-Unis dans la défense de la liberté en Europe (ils utilisent le concept d'intégration occidentale, Westintegration, alors que la partie française emploie le mot d'atlantisme, qui exprime l'idée d'une domination américaine jugée impé-

(1) La publication de ce volume est annoncée pour le début de l'année 2008. Le dernier volume, dédié à la classe de seconde, est prévu pour 2009.

(2) Les rencontres de Spire sont des rencontres d'historiens qui devaient contribuer à rapprocher les sociétés civiles des deux pays par l'examen des historiographies nationales et des manuels scolaires.

(3) Je remercie l'ancien inspecteur pédagogique régional et membre du Comité de pilotage Marcel Spisser, du rectorat de Strasbourg, pour le récit de cette divergence franco-allemande. 
rialiste). Au total, cette relative absence de difficultés traduit ce qu'Étienne François appelle « les vertus du bilatéral » (1), à savoir la tradition d'échanges féconds au niveau de la recherche entre la France et l'Allemagne, tradition qui s'inscrit dans des institutions de recherche telles que le Centre Marc Bloch à Berlin, la Mission historique française en Allemagne à Göttingen ou l'Institut historique allemand à Paris, et qui contribue à l'émergence de nouvelles générations d'historiens binationaux formés en partie dans l'autre pays.

Une fois le cahier des charges établi, les autorités de tutelle ont souhaité faire paraître en premier lieu le volume pour la classe de terminale. L'appel d'offres a été remporté par Nathan et Klett, seules maisons d'édition à s'être portées candidates. Spécialistes de l'édition scolaire, elles souhaitent occuper dès à présent un créneau qu'elles estiment devoir se développer rapidement dans un futur proche. La rédaction des chapitres a été confiée à une équipe franco-allemande de dix enseignants du secondaire, qui a travaillé en tandem sous la direction conjointe de deux auteurs expérimentés dans ce domaine, Guillaume Le Quintrec et Peter Geiss. Sous la pression des instances gouvernementales, tous les obstacles ont été levés en un temps record, notamment ceux qui étaient liés au fédéralisme allemand (2), pour permettre la parution de l'ouvrage en mai 2006, soit trois ans après le lancement du projet. Présentes à chaque étape, les autorités de tutelle ont exercé de fortes contraintes sur le comité de pilotage pour assurer l'aboutissement rapide de cette entreprise éditoriale inédite.

Tout en soulignant l'originalité du manuel franco-allemand, il convient de rappeler qu'il est aussi et surtout l'aboutissement d'une idée dont les racines intellectuelles remontent à l'entre-deux-guerres et du travail de réconciliation intellectuelle opéré après 1945 . Il symbolise l'émergence d'un milieu d'historiens franco-allemands partageant les mêmes avancées méthodologiques et historiographiques, mus par la volonté déconstruire une certaine histoire nationale.

(1) Étienne François, «Les vertus du bilatéral », Vingtième siècle. Revue d'histoire, $\mathrm{n}^{\circ} 71$, juillet-septembre 2001, pp. 91-95.

(2) Le défi en Allemagne était immense dans la mesure où chaque Land exerce ses prérogatives en matière d'éducation. Il a donc fallu harmoniser les programmes des seize Bundesländer. À cet égard, le rôle du plénipotentiaire de la République fédérale pour les affaires culturelles Peter Müller, ministre-président du Land de Sarre, a été primordial. 


\section{POUR UNE HISTOIRE TRANSNATIONALE, CROISÉE ET DÉNATIONALISÉE}

La réalisation du manuel franco-allemand a été saluée, à juste titre, comme une entreprise originale et inédite. Mais le succès de ce projet doit aussi se mesurer à sa capacité à convaincre les enseignants et les élèves de l'utiliser. Quel est l'intérêt pédagogique de cet instrument? En quoi est-il différent des manuels antérieurs?

\section{Les jeux d'échelles du manuel franco-allemand}

Les manuels franco-français classiques des classes de terminale constituent de bons bréviaires d'histoire des relations internationales (c'est-à-dire de la confrontation Est-Ouest) et d'histoire de France. Le manuel franco-allemand repose, quant à lui, sur un jeu d'échelles à trois niveaux qui sont en interaction permanente: le monde, l'Europe et le cadre national (France et Allemagne). La double logique qui traverse l'ouvrage est celle d'une histoire globale et croisée: le but est d'inciter les enseignants et les élèves à penser l'histoire dans un cadre dénationalisé, c'est-à-dire à pratiquer une histoire de l'Europe qui relève d'une perspective multilatérale. Cela signifie concrètement qu'on part de l'histoire globale pour arriver au cadre national et qu'on s'intéresse à la façon dont tel ou tel phénomène historique est perçu à l'étranger. L'exemple le plus significatif, qui reflète l'esprit du manuel, est la première partie, consacrée au bilan de la Seconde Guerre mondiale, dans laquelle sont traitées les «mémoires » de cette guerre. La perspective tout à la fois transnationale et diachronique permet l'historicisation de ces mémoires en montrant qu'on retrouve la même succession de phases dans de nombreux pays: à une étape d'héroïsation, destinée à construire au sein de la société le consensus indispensable à la reconstruction pacifique du pays, succède une phase de déconstruction du mythe "résistancialiste », couplée à une universalisation de la mémoire de la Shoah. Ensuite, le regard croisé proposé par le manuel permet de travailler sur les cas français et allemands en montrant les différences de chronologies et de problématiques entre les deux pays. On peut certes regretter l'absence du traitement des pays d'Europe de l'Est, où la mémoire de la Shoah entre en concurrence avec celle des crimes staliniens depuis le début des années 1990 (1), mais il y a là une possibilité offerte à l'enseignant d'exploiter vraiment son espace de

(1) Emmanuel Droit, " Le Goulag contre la Shoah dans l'Europe élargie », Vingtième Siècle. Revue d'histoire, $\mathrm{n}^{\circ}$ 94, avril-juin 2007, pp. 101-120. 
liberté pédagogique en pratiquant une histoire transnationale de l'Europe. En tout cas, la révolution copernicienne proposée par le manuel est bien la déconstruction de l'histoire nationale et sa réinsertion dans un cadre géographique plus large.

\section{Pour une histoire dénationalisée}

Le manuel franco-allemand d'histoire est incontestablement le produit de son temps, c'est-à-dire qu'il reflète les dernières tendances historiographiques. Il est en phase avec le temps de la mondialisation. Sa logique renvoie en effet au courant dit de l'histoire globale (Global history / Transnationale Geschichte) (1): cette histoire est centrée sur l'étude de phénomènes transnationaux comme les échanges économiques, migratoires, scientifiques, mais aussi des processus de convergence et de divergence entre les diverses régions du monde et en leur sein. La quatrième partie du manuel, consacrée aux transformations techniques, économiques, sociales et culturelles dans le monde depuis 1945, illustre bien l'abandon des perspectives nationales au profit d'études thématiques comparatives transnationales sur l'économie, la famille, la communication.

Cette histoire transnationale ne signifie pas l'abandon du cadre national, mais simplement l'insertion de la nation dans un cadre plus large. Elle permet de dénationaliser l'histoire de France et d'Allemagne en tant qu'histoire monumentale, « à la Lavisse »: en déconstruisant des entités qu'on a longtemps présentées comme typiquement allemandes ou françaises (le «Sonderweg » allemand, la fameuse « exception française »), ce manuel entend contribuer à éduquer des citoyens européens. Si la présence de faits concernant la RFA et la RDA aboutit à un allègement des pages consacrées à l'histoire de la France, l'élève est, en échange, très souvent invité à développer une perspective comparatiste trop fréquemment négligée dans l'enseignement secondaire. Au-delà de la traditionnelle mise en relief des différences et des ressemblances entre la France et l'Allemagne (par exemple dans les structures politiques et administratives), le comparatisme présente l'énorme avantage d'inciter l'élève à une pratique conceptualisée de l'histoire. Ainsi, la comparaison de la situation des étrangers et immigrés en France et en Allemagne depuis

(1) Voir à ce sujet l'ouvrage collectif de Gunilla Budd, Sebastian Conrad et Oliver Janz (dir.), Transnationale Geschichte. Themen, Tendenzen und Theorien, Göttingen, Vandenhoeck \& Ruprecht, 2006. 
1945 implique un travail préalable sur des concepts et des catégories, par exemple sur le droit de la nationalité. En effet, les notions se construisent dans le temps, elles ont une historicité qu'il s'agit de retracer. D'un autre côté, le comparatisme permet également de souligner le caractère parfois irréductiblement national de certaines notions. Ainsi, la comparaison des systèmes éducatifs conduit nécessairement à mettre en perspective le caractère radicalement différent de la Bildung allemande (1) par rapport à l'éducation telle qu'on l'entend en France. Comme le rappelle à juste titre É. François, « le comparatisme est une école à la fois de distanciation et de perspicacité » (2). En somme, l'approche comparatiste du manuel franco-allemand offre la possibilité d'apprendre autrement l'histoire nationale, de relativiser le cadre national d'analyse, bref de retrouver les dimensions transnationales de l'histoire de la France et de l'Allemagne.

\section{Les apports d'une didactique franco-allemande}

Au-delà des apports historiographiques les plus récents, les concepteurs et les auteurs du manuel franco-allemand ont relevé avec succès le défi de combiner les traditions didactiques propres aux deux pays. Du point de vue de sa présentation, le manuel franco-allemand d'histoire est très « français » dans sa conception. La page de droite est consacrée au texte de l'auteur tandis que la page de gauche est réservée aux divers documents. Mais on retrouve dans l'ouvrage le double souci allemand de rendre l'histoire vivante (avec l'introduction de jeux de rôles) et de la faire comprendre (par l'explication de la terminologie utilisée). Derrière le soin accordé aux définitions apparaît la volonté de déconstruire les concepts employés par les acteurs de l'histoire pour les rendre accessibles aux élèves. L'apport didactique français réside avant tout dans l'attention portée à l'espace, c'est-à-dire au lien entre histoire et territoire, qui se traduit dans le manuel par la présence de nombreuses cartes, alors que les manuels allemands se caractérisent par l'omniprésence de sources écrites, ce qui nous rappelle que l'histoire en Allemagne est intrinsèquement liée à la philosophie et à la philologie.

(1) Le mot Bildung (qui s'est fixé dans la langue allemande au début du $\mathrm{XIX}^{\mathrm{e}}$ siècle) renvoie par ses origines linguistiques à la création et à la formation de l'individu en tant qu'être humain perfectible. Voir Günther Dohmen, Bildung und Schule: Die Entstehung des deutschen Bildungsbegriffs und die Entwicklung seines Verhältnisses zur Schule, Weinheim, Beltz, 1964.

(2) Étienne François, «Les vertus du multilatéral », art. cit. 
Par ailleurs, ce manuel se caractérise par une grande richesse iconographique. L'importance de l'image en son sein est bien traduite par la remarque ironique d'un élève allemand du lycée franco-allemand de Fribourg-en-Brisgau: "Le manuel d'histoire franco-allemand est un livre type Stern, c'est-à-dire avec beaucoup d'images et peu de textes, à la différence de nos manuels allemands qui sont plutôt des livres type Spiegel, c'est-à-dire avec beaucoup de textes et peu d'images » (1). Au-delà de cette boutade, il est important de souligner l'originalité des images présentes dans le manuel franco-allemand par rapport à celles des manuels classiques: les enseignants français disposent grâce à lui de ressources iconographiques allemandes pour traiter des objets historiques comme le Plan Marshall ou la construction européenne. À travers ces images, l'enjeu est toujours de dénationaliser la vision qu'on peut avoir d'un phénomène ou d'une réalité historique. Il revient aux enseignants de ne pas uniquement considérer la ressource iconographique comme une simple illustration, mais bien comme une source à part entière, qui doit faire l'objet d'une analyse (2).

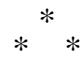

Depuis sa sortie, le manuel franco-allemand d'histoire constitue un succès éditorial, à la différence de l'Euromanuel publié au début des années 1990 (3). En Allemagne, l'éditeur Klett vient de lancer une sixième édition de l'ouvrage, qui s'est déjà vendu à 30000 exemplaires en l'espace de quelques mois. En France, Nathan est également très satisfait des ventes, qui s'élevaient également à 30000 volumes à l'automne 2006. Il est intéressant de noter que ce manuel a certes été acheté par des collectivités territoriales, mais qu'il s'est également vendu en librairie. Des citoyens dont la curiosité intellectuelle a été aiguisée par ce projet inédit ont donc acheté ce manuel qu'ils ont lu comme un simple livre d'histoire: le fait est suffisamment exceptionnel pour être relevé. Ce succès commercial est d'autant plus remarquable que le moment de la sortie du manuel était décalé par rapport au

(1) Entretien réalisé au lycée franco-allemand de Fribourg-en-Brisgau à l'occasion de la table ronde sur le manuel franco-allemand qui s'est tenue le 23 mai 2007. Der Spiegel et Der Stern sont deux grands hebdomadaires allemands d'information fondés après 1945. Le premier présente des enquêtes d'investigation plus fouillées que le second.

(2) Elisabeth Erdmann, « Apprendre l'histoire à l'aide d'images? Expériences scolaires et universitaires », in Marie-Christine Baquès, Annie Bruter, Nicole TutiauxGuillon (dir.), Pistes didactiques..., op. cit., pp. 285-300.

(3) Frédéric Delouche (dir.), Histoire de l'Europe, Paris, Hachette, 1997. 
cycle traditionnel de renouvellement des manuels dans le secondaire. En outre, on observe qu' un nombre non négligeable d'exemplaires produits dans un des deux pays a été acheté dans l'autre à destination des élèves des sections européennes et AbiBac. Enfin, le Luxembourg a également acheté ce manuel pour ses classes de terminale.

Le succès de cette entreprise inédite suscite l'intérêt d'autres pays, à commencer par l'Espagne, qui a manifesté son désir de participer, sous une forme encore à définir, à l'aventure du manuel commun. Mais cet intérêt dépasse le cadre de l'Europe. Dans l'espace AsiePacifique, la Corée et le Japon ont suivi avec la plus grande attention la gestation et l'évolution du projet (1). Des chaînes de télévision étrangères, sud-coréennes notamment, ont tourné cette année des reportages sur des classes utilisant le manuel franco-allemand et se font l'écho de cette nouvelle façon d'enseigner l'histoire.

En dépit de critiques sur son contenu formulées par d'éminents universitaires et experts des relations franco-allemandes (2), le manuel franco-allemand d'histoire est un succès commercial, mais également pédagogique. Son intérêt réside surtout dans son respect des spécificités des cultures nationales, c'est-à-dire dans la pluralité des interprétations qu'il propose: il ne présente pas un regard monolithique sur l'histoire, il respecte les divergences de points de vue, sur le rôle des États-Unis en Europe et dans le monde après 1945, sur le jugement à porter sur les expériences socialistes en Europe de l'Est... C'est ce regard croisé sur la façon dont l'Autre pense certains chapitres de l'histoire européenne et internationale qui lui donne sa nouveauté et son intérêt supplémentaire par rapport aux manuels classiques.

Emmanuel DROIT

Lycée International des Pontonniers (Strasbourg)

(1) Le manuel franco-allemand d'histoire a d'ailleurs son pendant en Asie avec le manuel « CJK » (China, Japan, Korea) qui traite de l'histoire moderne et contemporaine de ces trois pays d'Asie orientale. Voir Alain Delissen, «La nouvelle bataille des Falaises rouges? À propos du manuel commun "Chine-Corée-Japon" », Vingtième Siècle. Revue d'histoire, ${ }^{\circ}$ 94, avril-juin 2007, pp. 57-71.

(2) Alfred Grosser, « Le manuel franco-allemand: une déception », in La Croix, 26 juillet 2006. Sa principale critique est que ce manuel n'encourage pas les lycéens «à mener une vie engagée». Voir aussi le dossier coordonné par Ulrich Pfeil et Reiner Marcowitz dans la revue Dokumente. Zeitschrift für den deutsch-französischen Dialog, 5, 2006, pp. 53-102. 